\title{
Pubertad precoz en niñas: potencial impacto de la pandemia y el confinamiento por COVID-19
}

\author{
Precocious puberty in girls: potential impact of the COVID-19 pandemic and confinement
}

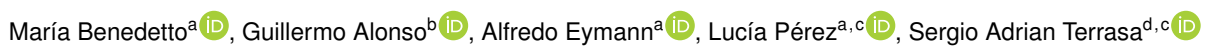

\begin{abstract}
Resumen
En este artículo, los autores revisan el concepto de pubertad precoz y los factores que pueden influenciar su desarrollo, en especial en las niñas y en el contexto de las medidas de aislamiento social preventivo y obligatorio durante periodos prolongados a causa de la actual pandemia por el SARS-CoV-2.
\end{abstract}

\begin{abstract}
In this article, the authors review the concept of precocious puberty and the factors that may influence its development, especially in girls and in the context of preventive and mandatory social isolation measures for prolonged periods of time due to the current SARS-CoV-2 pandemic.
\end{abstract}

Palabras clave: Pubertad Precoz, Pandemia de COVID-19, Confinamiento. Keywords: Precocious Puberty, COVID 19 Pandemic, Confinement.

Benedetto M, Alonso G, Eymann A, Pérez L y Terrasa SA. Pubertad precoz en niñas: potencial impacto de la pandemia y el confinamiento por COVID-19. Evid Actual Pract Ambul. 2021;24(3):e002160. Available from: https://dx.doi.org/10.51987/EVIDENCIA.V24I4.6974.

\section{¿A qué llamamos pubertad precoz?}

La pubertad es el resultado de un sistema neuroendócrino complejo, iniciado por mecanismos primarios no del todo conocidos y caracterizado por un incremento de liberación de gonadotrofinas $(\mathrm{GnRH})$ hipotalámicas ${ }^{1}$. Está relacionada con el desarrollo de los caracteres sexuales secundarios, la aceleración de la velocidad del crecimiento, la aparición de la menarquia (en las niñas) y la adquisición de la capacidad reproductiva ${ }^{2}$.

Los mecanismos fisiológicos vinculados a la reproducción implican un gran consumo energético, existiendo además una importante influencia genética en el inicio de la pubertad. Sin embargo, múltiples estudios han comunicado que el desarrollo puberal se ha vuelto cada vez más temprano en las últimas décadas, influido por factores ambientales como el peso, los hábitos dietarios, la actividad física, y la exposición a pantallas y químicos disruptores endócrinos ${ }^{3-5}$.

En las niñas se denomina pubertad precoz (PP) al desarrollo de caracteres sexuales secundarios antes de los ocho años de edad, al de menarca antes de los nueve años y, también, a la rápida progresión de los cambios puberales, aún cuando estos hayan comenzado luego de los ocho años de edad. La incidencia de PP se estima en $1 / 5.000$ a $1 / 10.000$ por año y es más frecuente en mujeres, con una relación mujeres/varones de 20:16.

Los casos de PP se pueden diferenciar entre aquellos dependientes de la hormona liberadora de la gonadotropina $(\mathrm{GnRH})$ y los independientes de esta hormona. La pubertad precoz de tipo central (PPC) es la más frecuente y ocurre en alrededor de $98 \%$ de los casos. Se debe a una activación prematura de la $\mathrm{GnRH}$, que puede ser inducida por tumores $u$ otros factores dependientes del sistema nervioso central o bien por factores no identificables (PPC idiopática) en el $90 \%$ de los casos. En cambio, la PP periférica depende de la producción de esteroides sexuales a nivel suprarrenal o gonadal, y más raramente, de la producción tumoral ectópica de sustancias con actividad gonadotrópica.

\section{Pubertad precoz y COVID-19}

Como ya fue mencionado en un artículo previo ${ }^{7}$, la pandemia de la enfermedad por coronavirus 2019 (COVID-19) ha tenido repercusiones sanitarias, sociales y económicas de gran alcance. A nuestro entender, una de las más importantes ha sido la interrupción de la presencialidad de los programas escolares con el fin de contener y mitigar la propagación de esta enfermedad. Así es como, durante el periodo de aislamiento preventivo y obligatorio (ASPO), las familias debieron realizar sus actividades habituales desde sus hogares, con el consiguiente aumento de su tiempo de exposición a pantallas, de conductas sedentarias, cambios en los hábitos alimentarios y de alteraciones en su ciclo sueñovigilia $^{8,9}$

Más allá de las consecuencias por todos conocidas, destacamos que un estudio un llevado a cabo en Italia en un Centro de Referencia de Trastornos Endócrinos -recientemente publicado por Stagi y col.-, documentó en forma retrospectiva un claro aumento en el número de diagnósticos de PPC durante la pandemia respecto de los realizados en la misma institución durante los cinco años anteriores (37 pacientes durante la pandemia, vs. un total de 89 durante los cinco años previos, $p<0,0005$ ), lo que podría estar vinculado a ciertos cambios de hábitos de vida (aumento del tiempo frente a dispositivos con pantallas, aumento de la adiposidad, disminución de la actividad física, cambios en los patrones de sueño-vigilia, etc. ${ }^{1}$.

Si bien no quedan claros los mecanismos causales, podría pensarse que la radiación lumínica de las pantallas de los actuales dispositivos podrían infuir en algunos procesos vinculados a la regulación de los ciclos circadianos a través de circuitos a nivel de la glándula pineal (ciclos luz/oscuridad) ${ }^{10}$. Por otro lado, existen también antecedentes bibliográficos que indican que los procesos de adopción acelerarían los procesos del desarrollo puberal. Una posible explicación de esta aceleración en el desarrollo puberal podría estar dada por el estrés resultante de los cambios ambientales consecutivos a la efectivización del proceso de adopción, y también estar vinculados a las mejoras nutricionales a las que se han visto expuestas estas niñas una vez ingresadas al nuevo

\footnotetext{
a Servicio de Clínica Pediátrica, Hospital Italiano de Buenos Aires. maria.benedetto@hospitalitaliano.org.ar, alfredo.eymann@hospitalitaliano.org.ar,

b Sección Endocrinología, Servicio de Clínica Pediátrica, Hospital Italiano de Buenos Aires. guillermo.alonso@hospitalitaliano.org.ar

c Departamento de Investigación, Hospital Italiano de Buenos Aires. lucia.perez@hospitalitaliano.org.ar, sergio.terrasa@hospitalitaliano.org.ar

d Servicio de Medicina Familiar y Comunitaria, Hospital Italiano de Buenos Aires.
} 
hogar, ya que el aumento de la cantidad de su tejido graso habría actuado como un activador de los sistemas endocrinos que regulan la pubertad ${ }^{11}$.
La Figura 1 muestra un modelo hipotético para tratar de explicar el potencial aumento de la incidencia de PP en niñas en el contexto de las medidas de aislamiento social durante la pandemia de COVID-19.

Figura 1. Modelo hipotético de la influencia del cierre de las actividades escolares presenciales durante la pandemia por el SARSCoV-2 y la velocidad del desarrollo puberal de las niñas. Fuente: elaboración propia de los autores. Nota: ASPO: Aislamiento Social Preventivo y Obligatorio.

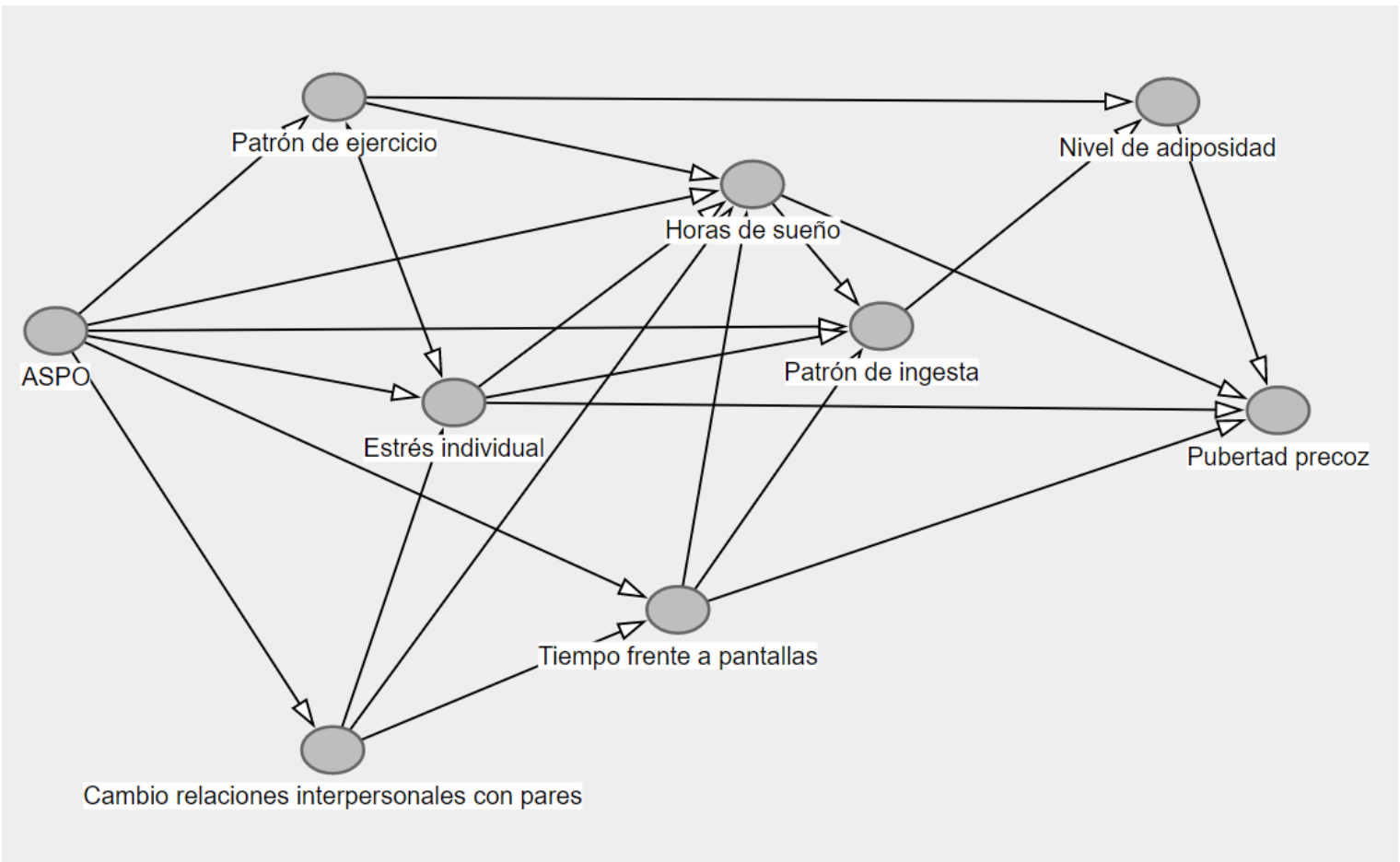

A pesar de que no contamos con información local al respecto, destacamos que los resultados comunicados por Stagi y col. ${ }^{1}$ son coincidentes con la percepción de los profesionales de la Sección Endocrinología del Servicio de Pediatría del Hospital Italiano de Buenos Aires.

Dado lo prolongado que ha sido el confinamiento domiciliario de los niños y las niñas de Argentina durante 2020, consideramos relevante investigar en forma sistemática si hubo cambios en la incidencia de PP en el contexto de dicho aislamiento, que ha aumentado el tiempo de exposición a pantallas, modificado los hábitos alimentarios, los ciclos sueño-vigilia y el índice de masa corporal de las niñas a nuestro cuidado ${ }^{3}$. Por esta razón, quienes firmamos este artículo estamos trabajando en el diseño de una investigación para objetivar en nuestra institución -un Hospital Académico que atiende pacientes domiciliadas en el Área Metropolitana de Buenos Aires- las modificaciones en la incidencia de PP ocurridas durante el confinamiento domiciliario al que se vieron obligadas las niñas desde el comienzo de la pandemia de COVID-19.

Para concluir, recordamos que durante el último siglo y medio hemos observado el adelantamiento de la menarca en las mujeres (a los 17 años de edad en 1850, a los 13 años en 1950 y a los 12,5 años en la actualidad) ${ }^{12}$. Aunque resulte difícil establecer inferencias causales respecto de la influencia del reciente confinamiento domiciliario sobre la aceleración de los procesos de maduración sexual, de constatarse también a nivel local este aumento en la incidencia de PP, los médicos de atención primaria podrán tener mayor alerta para detectar, de la manera más temprana posible, eventuales aceleraciones en la maduración sexual que puedan recibir tratamiento en forma oportuna.

Recibido el 20/09/2021 Aceptado el 24/09/2021 y Publicado el 29/09/2021.

\section{Referencias}

1. Stagi S, De-Masi S, Bencini E, et al. Increased incidence of precocious and accelerated puberty in females during and after the Italian lockdown for the coronavirus 2019 (COVID-19) pandemic. Ital J Pediatr. 2020;46(1):165-165. Available from: 10.1186/s13052-020-00931-3.

2. Bouvattier C. Pubertad normal. Pubertad precoz y retraso puberal. EMC - Ginecología-Obstetricia. 2017;53(2):1-11. Available from: 10.1016/ S1283-081X(17)84282-X

3. Martínez-Aedo-Ollero MJ, Godoy-Molina E. Pubertad precoz y variantes de la normalidad; 2019. Available from: https://www.aeped.es/sites/default/ files/documentos/15 pubertadp.pdf. 
4. Teilmann G, Boas M, Petersen JH, et al. Early Pituitary-Gonadal Activation before Clinical Signs of Puberty in 5- to 8-Year-Old Adopted Girls: A Study of 99 Foreign Adopted Girls and 93 Controls. J Clin Endocrinol Metab. 2007;92:2538-2582. Available from: 10.1210/jc.2006-2096.

5. Pietrobelli A, Pecoraro L, Ferruzzi A, et al. Effects of COVID-19 Lockdown on Lifestyle Behaviors in Children with Obesity Living in Verona, Italy: A Longitudinal Study. Obesity (Silver Spring). 2020;28(8):1382-1387. Available from: 10.1002/oby.22861

6. Brito VN, Spinola-Castro AM, Kochi C, et al. Central precocious puberty: revisiting the diagnosis and therapeutic management. Arch Endocrinol Metab. 2016;60(2):163-72. Available from: 10.1590/2359-3997000000144.

7. Perman G, Puga C, Ricci I, et al. Daños colaterales de la pandemia por COVID-19 ¿Consecuencias inevitables? Rev Hosp Ital BAires. 2020;40(4):000. Available from: https://www1.hospitalitaliano.org.ar/educacion/revista/index.php?contenido=ver_articulo.php\&id_articulo=115688.

8. Verzani M, Bizzarri C, Chioma L, et al. Impact of COVID-19 pandemic lockdown on early onset of puberty: experience of an Italian tertiary center. Ital J Pediatr. 2021;47(1):52. Available from: 10.1186/s13052-021-01015-6.

9. Gavela-Pérez T, Garcés C, Navarro-Sánchez P, et al. Earlier menarcheal age in Spanish girls is related with an increase in body mass index between pre-pubertal school age and adolescence. Pediatr Obes. 2015;10(6):410-415. Available from: 10.1111/ijpo.277.

10. Hysing M, Pallesen S, Stormark KM, et al. Sleep and use of electronic devices in adolescence: results from a large population-based study. BMJ Open. 2015;5(1):6748-6748. Available from: 10.1136/bmjopen-2014-006748.

11. Hayes P. International adoption, early puberty, and underrecorded age. Pediatrics. 2013;131(6):1029-1060. Available from: 10.1542/peds.2013 0232.

12. Eckert-Lind C, Busch AS, Petersen JH. Worldwide Secular Trends in Age at Pubertal Onset Assessed by Breast Development Among Girls: A Systematic Review and Meta-analysis. JAMA Pediatr. 2020;174(4):195881-195881. 